\title{
Direct Spherical Harmonic Transform of a Triangulated Mesh
}

\author{
M. MOUSA \\ R. CHAINE \\ S. AKKOUCHE
}

L.I.R.I.S : Lyon Research Center for Images and Intelligent Information Systems

Bâtiment Nautibus, 8 boulevard Niels Bohr

69622 Villeurbanne Cedex, FRANCE

\{mmousa, rchaine, sakkouch\}@liris.cnrs.fr

\begin{abstract}
Spherical harmonic transform plays an important role in research in shape description. Current computation methods involve expensive voxelization, and are prone to numerical errors associated with the size of the voxels. This paper describes a fast and accurate technique for computing spherical harmonic coefficients directly from the description of the mesh.
\end{abstract}

\section{Introduction}

If $M$ is a closed embedded surface in 3-space then there is a solid volume $V$ bounded by $M$. Fixing a point $P$, one can consider the intersection of $V$ with a sphere $S_{r}$ of radius $r$ around $P$. In general, we choose $P$ at the centroid of the object. The intersection $M_{r}=V \cap S_{r}$ is a region of the sphere. The characteristic function $\chi_{r}$ of this region ( 1 for points inside, 0 for those outside) can be approximated by a sum of spherical harmonics $Y_{l}^{m}(\theta, \varphi)$ in the spherical coordinates. Theoretically, the expansion of $\chi_{r}$ in terms of spherical harmonics is written as:

$$
\chi_{r}(\theta, \varphi)=\sum_{l=0}^{\infty} \sum_{|m| \leq l} c_{l m}^{r} Y_{l}^{m}(\theta, \varphi)
$$

This expansion corresponds to a frequency-based decomposition of $\chi_{r}$. In practice, since higher order coefficients $c_{l m}^{r}$ correspond to finer details of the objects (maybe noise), we limit this summation to a bandwidth denoted as $b w$ :

$$
\chi_{r}(\theta, \varphi) \approx \sum_{l=0}^{b w} \sum_{|m| \leq l} c_{l m}^{r} Y_{l}^{m}(\theta, \varphi)
$$

A collection of such approximations of the characteristic function $\chi_{r}$, for several values of $r$, has proved to be useful as shape-descriptors for searching and identifying $3 \mathrm{D}$ objects $[3,8]$.

In this paper, we show how to compute the coefficients of the spherical harmonic representation of $\chi_{r}$ quickly and accurately when the surface $M$ is given by a polyhedral mesh. The computation of each coefficient $c_{l m}^{r}$ has an $O(n)$ complexity, where $n$ is the number of triangles of the mesh.

Standard algorithms $[3,6,7]$ compute a voxelization of $V$ and then use this discrete approximation to find the coefficients. The discretization introduces errors in the integrations needed to compute the harmonic coefficients. In contrast, our method does not require this discrete approximation of $V$ to calculate the integration. The numerical quality of the voxelized methods can approximate ours when the voxel grid is chosen small enough. Mesh voxelization can be computed in a time that is approximately linear in the number of voxels that meet the mesh [5]. The integration of the function over these voxels is then linear in the number of voxels. Our algorithm is linear in the number of mesh triangle. Thus if the voxelization generates approximately a constant number of voxels per triangle, the two methods have identical asymptotic running times.

This paper is organized as follows. The next section proposes a means to decompose the spherical harmonic transform of $\chi_{r}$ into a sum of more elementary spherical harmonic transforms computed on a set of spherical triangles. Section 3 gives a theoretical discussion to compute the spherical harmonic coefficients. Section 4 presents two methods to compute this harmonic coefficient for a single spherical triangle. Finally, section 5 gives some experimental results followed by the conclusion in section 6 . 

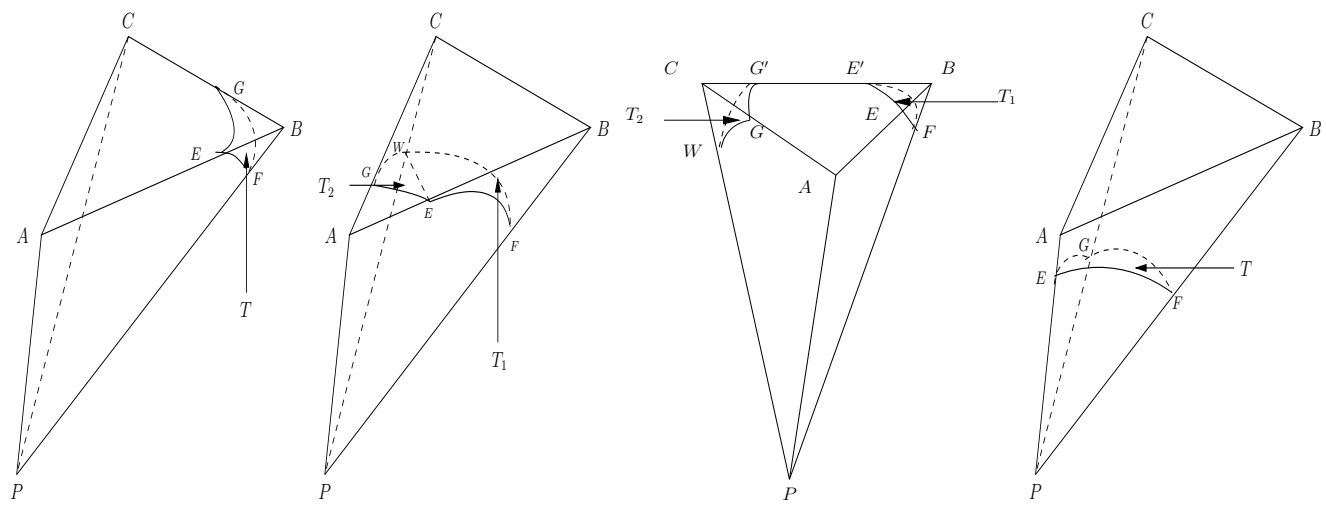

(a) $A$ and $C$ are inside (b) $A$ is inside the (c) $A$ is inside the sphere $S_{r}$ (d) $A, B$ and $C$ are the sphere $S_{r} . E G$ is sphere $S_{r} . E G$ is the and $S_{r}$ intersects the edge $B C$. outside the sphere. All the only non geodesic only non geodesic arc. $E E^{\prime}$ and $G G^{\prime}$ are the only non arcs are geodesics arc. geodesic arcs.

Figure 1: intersections of the sphere $S_{r}$ and a tetrahedron $H$.

\section{Details}

The volume $V$ can be defined as a union of tetrahedra, one from each triangle of the mesh to the point $P$. The triangles of the mesh are assumed to be oriented consistently, i.e., for two neighboring triangles, the shared edge has two different directions. If the normal of a triangle and $P$ are in the opposite side with respect to the triangle, the corresponding tetrahedron is said to be positive, and negative otherwise.

Let $\left\{H_{k}, k=1, \ldots, n\right\}$ denote the set of signed tetrahedra. We split $\left\{H_{k}\right\}$ into two subsets $\left\{H_{i}^{+}\right\}$and $\left\{H_{j}^{-}\right\}$according to the signs of the tetrahedra. The signed volume enclosed by the triangulated mesh can be represented as :

$$
V=\left(\bigcup_{i} H_{i}^{+}\right)-\left(\bigcup_{j} H_{j}^{-}\right)
$$

More formally, this means that a point is defined to be in $V$ if the sum of the signs of all tetrahedra that it occupies is positive. Zhang and Chen [10] have shown that this decomposition can be used to calculate global volumetric moments on $V$ as a sum of elementary volumetric moments computed on each tetrahedron of the decomposition.

$$
\operatorname{moment}(V)=\sum_{k} \operatorname{sign}\left(H_{k}\right) \operatorname{moment}\left(H_{k}\right)
$$

We extend this property to directly compute the spherical harmonic transform of the triangulated mesh $M$. To compute the spherical harmonic coefficients of $\chi_{r}$, we observe that the set $M_{r}=V \cap S_{r}$ is a signed union of spherical triangles $\left\{T_{k}, \quad k=1, \ldots, s_{n}\right\}$ arising from the intersections of the sphere $S_{r}$ with the tetrahedra of $V$. Let $\chi_{T_{k}}$ denote the characteristic function of $T_{k}$. Therefore $\chi_{r}$ can be represented as :

$$
\chi_{r}(\theta, \varphi)=\sum_{k} \operatorname{sign}\left(T_{k}\right) \chi_{T_{k}}(\theta, \varphi)
$$

and

$$
c_{l, m}^{r}=\left.\sum_{k} \operatorname{sign}\left(T_{k}\right) c_{l, m}^{r}\right|_{T_{k}}
$$

The decomposition of $M_{r}$ into a signed union of spherical triangles is obtained from the intersection of $S_{r}$ with the set of signed tetrahedra $\left\{H_{k}\right\}$. There are four general cases for the intersection of $S_{r}$ and the tetrahedron $H=P A B C$ (recall that $P$ is the center of the sphere).

- If $A, B$ and $C$ are inside $S_{r}$, then there is no intersection.

- If one of $A, B$ and $C$ is outside $S_{r}$, then $S_{r}$ intersects the edges of $H$ at three points forming a spherical triangle $T$ (figure $1(\mathrm{a})$ ). 
- If two of $A, B$ and $C$ are outside $S_{r}$, then $S_{r}$ intersects the edges of $H$ at four or six points forming a union of two spherical triangles $T_{1} \cup T_{2}$ (figure 1(b) and 1(c)).

- If $A, B$ and $C$ are all outside $S_{r}$, then $S_{r}$ intersects the edges of $H$ at three points forming a spherical triangle $T$ (figure 1(d)). An interior part of the triangle $A B C$ may be inside $S_{r}$. Then the spherical triangle $T$ does not entirely lie inside $H$ (figure 2(a)). To deal with that case, let $Q$ be a point on $A B C$ lying inside $S_{r}$. Then the tetrahedron $H$ can be seen as the union of the tetrahedra $P A B Q$, $P B C Q$ and $P C A Q$ (figure 2(b)) which can be treated as in the second case (figure 1(b) or 1(c)). Note that those tetrahedra raise spherical triangles with at most one non geodesic arc for each.

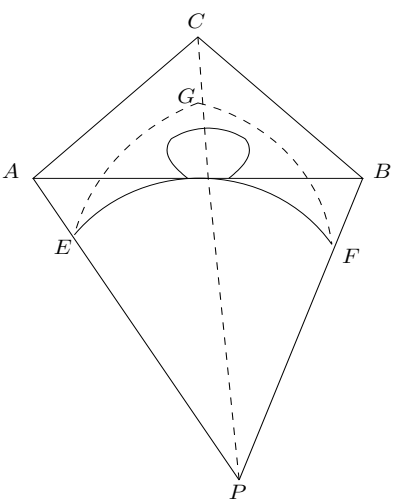

(a)

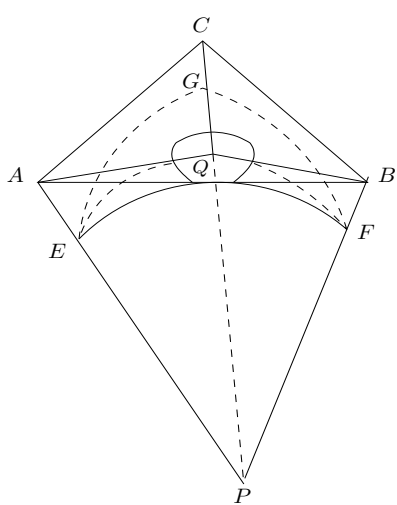

(b)

Figure 2: particular case of a triangle $A B C$ whose vertices are outside the sphere but not the entire triangle.

The sign of a spherical triangle is inherited from the tetrahedron it comes from. We compute the spherical harmonic coefficients for the characteristic function of a single spherical triangle $E F G$, and then sum the results. The details of the summation process are described in the next section.

\section{The coefficients for a single spherical triangle}

In this section, we describe the calculation of the harmonic coefficients over a single spherical triangle $T$. We first describe it for general spherical triangles and then address some special cases.

\subsection{General case}

To find the spherical harmonic coefficient $\left.c_{l, m}^{r}\right|_{T}$ for the characteristic function $\chi_{T}$, we must compute:

$$
\left.c_{l, m}^{r}\right|_{T}=\iint_{\mathbb{S}^{2}} \chi_{T}(\theta, \varphi) \bar{Y}_{l}^{m}(\theta, \varphi) \sin (\theta) d \theta d \varphi
$$

$Y_{l}^{m}$ is the spherical harmonic of degree $l$ and order $m$ where $l \geq 0$ and $m \leq|l|, \theta \in\left[\begin{array}{ll}0 & \pi\end{array}\right]$ is the polar

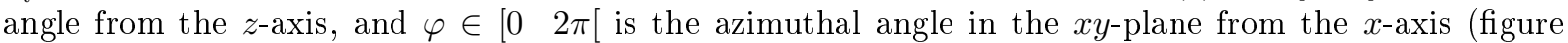
3). The expression of $\left.c_{l, m}^{r}\right|_{T}$ is the projection of $\chi_{T}$ on $Y_{l}^{m}$ since the spherical harmonics are a set of orthogonal functions. Recall that $Y_{l}^{m}(\theta, \varphi)$ is defined as :

$$
Y_{l}^{m}(\theta, \varphi)=k_{l m} P_{l}^{m}(\cos \theta) e^{i m \varphi}
$$

$k_{l m}$ is a constant depending on $l$ and $m$, and $P_{l}^{m}$ is the associated Legendre Polynomial [9]. For more details about spherical harmonics see $[1,4]$.

The expression to be integrated is nonzero only for points in $E F G$, so $\left.c_{l, m}^{r}\right|_{T}$ simplifies to

$$
\left.c_{l, m}^{r}\right|_{T}=\iint_{T} \bar{Y}_{l}^{m}(\theta, \varphi) \sin (\theta) d \theta d \varphi
$$




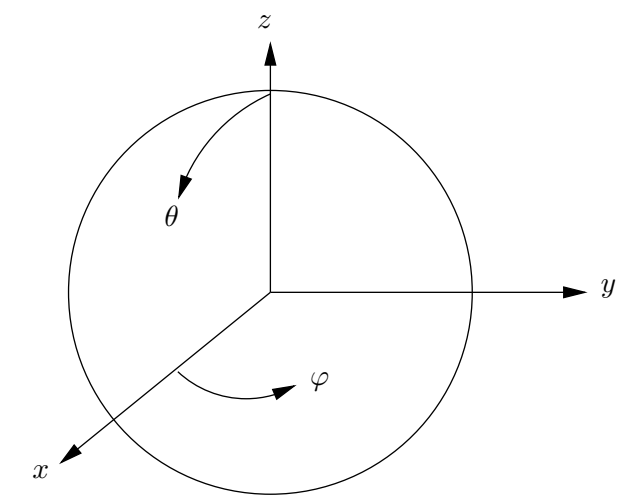

Figure 3: direction of $\theta$ and $\varphi$ in the spherical coordinate

By taking into account the bounds of $\theta$ and $\varphi$, the integral becomes

$$
\left.c_{l, m}^{r}\right|_{T}=\int_{\varphi_{1}}^{\varphi_{2}} \int_{\theta_{1}(\varphi)}^{\theta_{2}(\varphi)} \bar{Y}_{l}^{m}(\theta, \varphi) \sin (\theta) d \theta d \varphi
$$

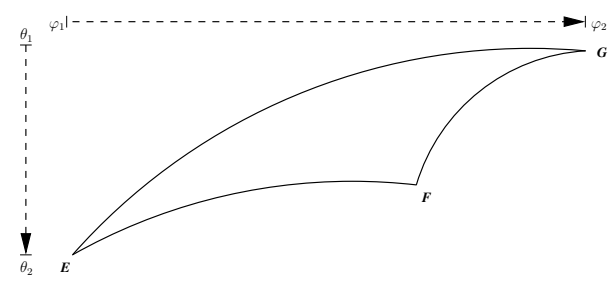

(a)

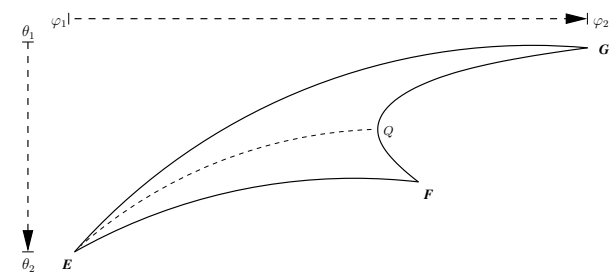

(b)

Figure 4: the $\theta, \varphi$ bounds for the spherical triangle $T$

$\varphi_{1}$ and $\varphi_{2}$ denote the bounds for $\varphi$ for the spherical triangle $T$ (Figure 4(a)). The inner integration corresponds to the integration along the geodesic path from $\theta_{1}(\varphi)$ to $\theta_{2}(\varphi)$. The relations $\theta_{1}(\varphi)$ and $\theta_{2}(\varphi)$ can be determined from the equations of the arcs of the spherical triangle (intersection between a plane and $S_{r}$ ). However, these relations are not linear and they depend mainly on the dimension and orientation of the spherical triangle $E F G$.

Note that if $F G$ is not a geodesic arc and that we meet the situation described in figure 4(b), then the inner integration will be incorrect. In this case, the spherical triangle can be split along a geodesic arc into two spherical triangles (with at most one non geodesic arc on each) and on which we can calculate the harmonic coefficient as in figure $4(\mathrm{a})$.

The harmonic coefficients $\left.c_{l, m}^{r}\right|_{T}$ are complex. They are related to each other by the following relation:

$$
\left.c_{l,-m}^{r}\right|_{T}=(-1)^{m} \overline{\left.c_{l, m}^{r}\right|_{T}}
$$

This allows one to do half as much work as would be otherwise necessary.

$$
(l-m) P_{l}^{m}(x)=x(2 l-1) P_{l-1}^{m}(x)-(l+m-1) P_{l-2}^{m}(x)
$$

Note that the recurrence relation of Legendre polynomials defined in equation 12 cannot be turned into a simple recurrence relation on spherical harmonic coefficients. This is not surprising since it insures that higher order harmonic coefficients cannot be expressed in terms of lower order harmonic coefficients.

\subsection{Special spherical triangles}

In this subsection, we describe three special cases of the spherical triangle. In the first case, the spherical triangle passes across the great circle $\varphi=0$ (figure $5(\mathrm{a})$ ). The bounds of $\varphi$ are not in correct order if one 
integrates from $\varphi_{1}=\min (\varphi)$ to $\varphi_{2}=\max (\varphi)$. So we reverse the bounds and integrate as follows:

$$
\left.c_{l, m}^{r}\right|_{T}=\int_{\varphi_{2}}^{2 \pi} \int_{\theta_{1}(\varphi)}^{\theta_{2}(\varphi)} \bar{Y}_{l}^{m}(\theta, \varphi) \sin (\theta) d \theta d \varphi+\int_{0}^{\varphi_{1}} \int_{\theta_{1}(\varphi)}^{\theta_{2}(\varphi)} \bar{Y}_{l}^{m}(\theta, \varphi) \sin (\theta) d \theta d \varphi
$$

In the second case, the spherical triangle contains the north or south poles ( $\theta$ equals 0 or $\pi$ respectively). The bounds of $\theta$ may lead to incorrect results. Without loss of generality let us consider a spherical triangle containing the north pole $N$. We divide the triangle $T=E F G$ into three spherical triangles $T_{1}=N E F, T_{2}=N F G$ and $T_{3}=N G E$ and then compute the sum of the integrations over each spherical triangle individually (Figure 5(b)).

In rare cases, the spherical triangle can be degenerate. This case is raised by the intersection of a degenerate tetrahedron (i.e. it has a zero volume) and the sphere. The spherical triangle in this case is reduced to a geodesic arc on the sphere. So, the surface integration on the sphere for that arc is $0\left(\theta_{1}(\varphi)=\theta_{2}(\varphi)\right.$ in equation 10). This is trivial since the volume of this tetrahedron is zero and volumetrically it does not contribute to the signed volume of the model.

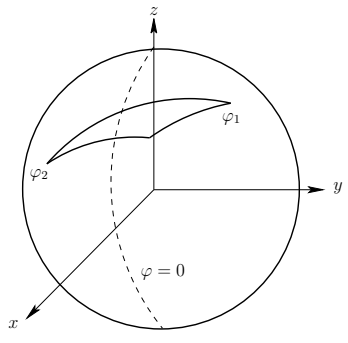

(a)

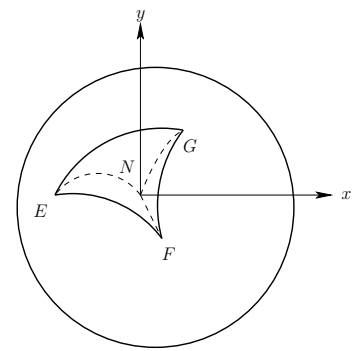

Figure 5: two special cases for $\theta$ and $\varphi$.

\section{Evaluating the Integration}

In this section, we propose two alternatives to avoid the nonlinear relationship between $\theta$ and $\varphi$. The first one is based on symbolic integration using any auxiliary library that supports symbolic integrations, like MATLAB or MATHEMATICA. The second one is based on numerical estimation of the integral.

\subsection{Symbolic Integration}

Actually, as far as we know, symbolic packages do not permit to find the final symbolic form of the integration expressed in equation 10 with respect to the relation between $\theta$ and $\varphi$. However, we can calculate the symbolic form of integration :

$$
\iint \bar{Y}_{l}^{m}(\theta, \varphi) \sin (\theta) d \theta d \varphi
$$

without the bounds of $\theta$ and $\varphi$. Then, we approximate the spherical triangle to vertical slices and evaluate the obtained formulae with the bound of each slice.

Consider without loss of generality that the spherical triangle $E F G$ is such that $\varphi_{E} \leq \varphi_{F} \leq \varphi_{G}$ and $\theta_{E} \geq \theta_{F}$, as shown in figure 6 . Therefore, the integral that computes $\left.C_{l, m}^{r}\right|_{T}$ can be divided into two subintegrals $I_{1}$ and $I_{2}$ :

$$
\left.C_{l, m}^{r}\right|_{T}=I_{1}+I_{2}
$$

where $I_{1}$ and $I_{2}$ are as follows :

$$
\begin{aligned}
& I_{1}=\int_{\varphi_{E}}^{\varphi_{F}} \int_{\theta_{E G(\varphi)}}^{\theta_{E F}(\varphi)} \bar{Y}_{l}^{m}(\theta, \varphi) \sin (\theta) d \theta d \varphi \\
& I_{2}=\int_{\varphi_{F}}^{\varphi_{G}} \int_{\theta_{E G}(\varphi)}^{\theta_{F G}(\varphi)} \bar{Y}_{l}^{m}(\theta, \varphi) \sin (\theta) d \theta d \varphi
\end{aligned}
$$




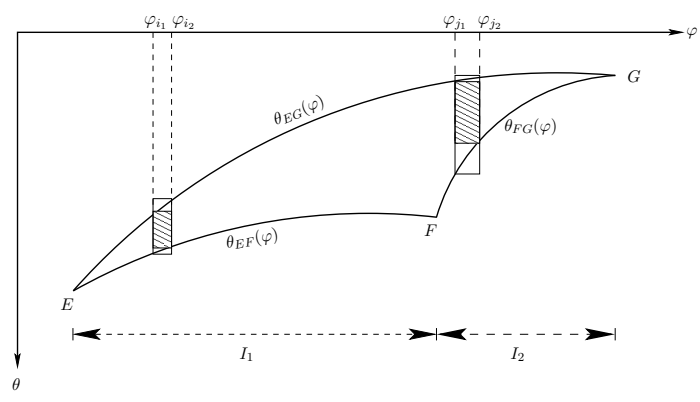

Figure 6: discretization of the spherical triangle into vertical slices

\begin{tabular}{|c|c|c|}
\hline$(l, m)$ & $\theta$ part & $\varphi$ part \\
\hline$(0,0)$ & $-\cos (\theta)$ & $\varphi$ \\
$(1,0)$ & $-1 / 4 * \cos (2 * \theta)$ & $\varphi$ \\
$(1,1)$ & $1 / 2 * \operatorname{sign}(\sin (\theta)) *(\cos (\theta) * \sin (\theta)-\theta)$ & $i e^{-i \varphi}$ \\
$(2,0)$ & $-1 / 8 * \cos (3 * \theta)+1 / 8 * \cos (\theta)$ & $\varphi$ \\
$(2,1)$ & $-1 / 8 *(2-2 * \cos (2 * \theta))^{(3 / 2)}$ & $i e^{-i \varphi}$ \\
$(2,2)$ & $-3 * \cos (\theta)+\cos (\theta)^{3}$ & $i e^{-2 i \varphi} / 2$ \\
\hline
\end{tabular}

Table 1: examples of integration of $\theta$ and $\varphi$ parts

$\theta_{u v}(\varphi)$ is the relation between $\theta$ and $\varphi$ on the boundary $u v$ of the considered spherical triangle. Splitting each of $I_{1}, I_{2}$ into stepwise subintegrals according to $\left\{\varphi_{i_{k}}\right\}_{k=0}^{s}$ and $\left\{\varphi_{j_{k}}\right\}_{k=0}^{t}$ respectively, we get:

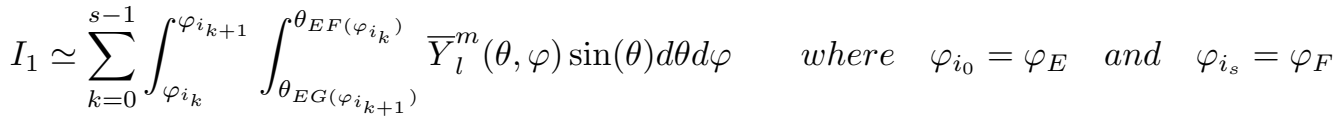

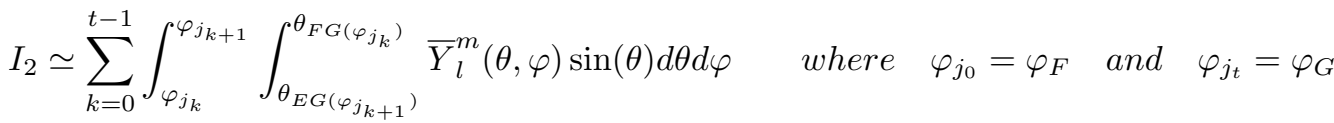

We discretize the spherical triangle into small enough vertical slices and integrate over each one separately, as shown in figure 6. Of course, the accuracy depends on the number of slices. Practically, this number can be chosen heuristically by regarding the increase (or decrease) of $\theta$ with respect to the increasing of $\varphi$. For example, if $\Delta \varphi \ll \Delta \theta$, the number of steps is taken according to $\Delta \theta$ and vice versa, where $\Delta \varphi=\max \varphi-\min \varphi$ and $\Delta \theta=\max \theta-\min \theta$.

On the other hand, the variables $\theta$ and $\varphi$ are separated in $Y_{l}^{m}(\theta, \varphi)$ (see equation 8). Considering that $\theta$ and $\varphi$ are independent, the integration can be done for each one separately. Therefore the integration can be written as:

$$
\iint \bar{Y}_{l}^{m} \sin (\theta) d \theta d \varphi=k_{l m}\left(\int P_{l}^{m}(\cos \theta) \sin \theta d \theta\right)\left(\int e^{-i m \varphi} d \varphi\right)
$$

Therefore, the integration of $\theta$ and $\varphi$ parts can be done symbolically using MATLAB and stocked in an external file to be evaluated later. The integration of $\varphi$ part is easy. It is equal to $i e^{-i m \varphi} / \mathrm{m}$ for $m \neq 0$, and $\varphi$ for $m=0$. The integration of $\theta$ part is more complicated than that of $\varphi$ part. Symbolic integrations used for lower order spherical harmonic coefficients are shown in table 1.

Finally, the symbolic formulae are evaluated for each slice using its $\theta, \varphi$ bounds, as shown in equations 18 and 19.

The symbolic integration has the following advantages:

- the formulae are exact,

- the symbolic formulae are stocked offline, so we maintain the execution time,

- execution time is faster than that of the numerical estimation described in next subsection, since it is only an inline evaluation of coded formulae with respect to $\theta$ and $\varphi$ bounds. 


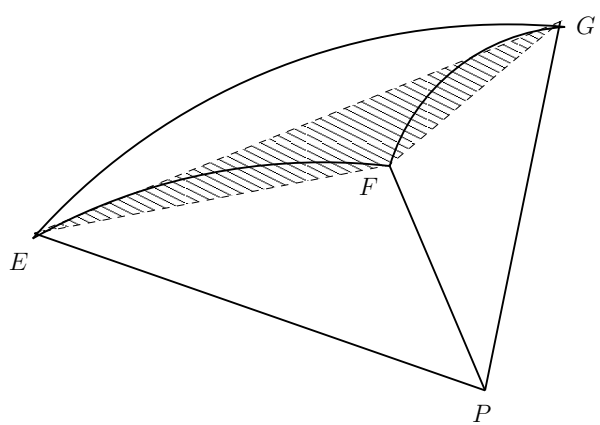

Figure 7: a spherical triangle and its corresponding euclidean triangle.

The symbolic integration also has some disadvantages:

- it neglects the exact dependency between $\theta$ and $\varphi$,

- it can not calculate the integrations of high order spherical harmonics because of the limitation of the available symbolic libraries (MATLAB can not compute integrations for the harmonics of order greater than 27),

- it is not trivial to define an explicit method that controls the level of division of the spherical triangle into small slices.

\subsection{Numerical Estimation}

A spherical triangle $E F G$ bounded by geodesic arcs can be parametrized by the euclidean triangle sharing the same vertices (figure 7). If $A B C$ is a triangle of the mesh, then the cone from the origin $P$ that is subtended by $A B C$ intersects the sphere in a spherical triangle $E F G$ with geodesic edges. Radial projection from $A B C$ to $E F G$ is a parameterization of this triangle, and can be used to integrate any function over the triangle. The function we want to integrate is :

$$
\chi \bar{Y}_{l}^{m}(\theta, \varphi)
$$

where $\chi$, for each point of $E F G$, is one if the point is between the triangle $A B C$ and the origin, and zero otherwise. In fact, each point $Q$ on the euclidean triangle $E F G$ can be written as:

$$
Q=\lambda E+\beta F+(1-\lambda-\beta) G \quad 0 \leq \lambda \leq 1 \quad 0 \leq \beta \leq 1 \quad 0 \leq \lambda+\beta \leq 1
$$

We can parametrize $(\theta, \varphi)$ by $(\lambda, \beta)$ using the conversion from the cartesian to spherical coordinates:

$$
\begin{aligned}
& \theta(\lambda, \beta)=\arctan \frac{\sqrt{x(\lambda, \beta)^{2}+y(\lambda, \beta)^{2}}}{z(\lambda, \beta)} \\
& \varphi(\lambda, \beta)=\arctan \frac{y(\lambda, \beta)}{x(\lambda, \beta)}
\end{aligned}
$$

Therefore, the integration over a spherical triangle bounded by geodesic arcs becomes:

$$
\left.c_{l, m}^{r}\right|_{T}=\int_{0}^{1} \int_{0}^{1} \bar{Y}_{l}^{m}(\theta(\lambda, \beta), \varphi(\lambda, \beta)) \sin \theta(\lambda, \beta) J\left[\frac{\theta, \varphi}{\lambda, \beta}\right] d \lambda d \beta
$$

where $J\left[\frac{\theta, \varphi}{\lambda, \beta}\right]$ is the Jacobian of $(\theta, \varphi)$ with respect to $(\lambda, \beta)$. Equation (25) can be evaluated using one of numerical integrations implemented in any scientific library. The examples presented in this article were evaluated using the plain Monte Carlo integration implemented in the GNU Scientific Library, GSL [2] with $10^{3}$ as the size of the iteration space.

The numerical estimation has the following advantages:

- it need not partition the spherical triangle, so its accuracy is more than the first one, 
- it maintains implicitly the dependency between $\theta$ and $\varphi$,

- it can compute high order harmonic coefficients,

However, its main drawback is that it is not fast because of the many numerical parameters (error bound, number of iterations...).

The numerical estimation described in this section can handle the case of the spherical triangle whose one boundary is not geodesic but also spherical triangles that miss an interior part (figure 2(a)) without splitting it into smaller ones. In fact, numerical integrations are achieved by evaluating the function at sample points that lie on the region of interest. The test of inside/outside the tetrahedron is what's done by the function $\chi$, and in practice this means simply checking each sample and throwing out those which lie outside the tetrahedron, and evaluating $\bar{Y}(\theta, \varphi)$ on the others and summing, and then dividing by the total count of both accepted and rejected samples.

From the advantages and disadvantages of the symbolic integration and numerical estimation, we prefer the numerical estimation to the symbolic integration.

\section{$5 \quad$ Experimental Results}

We have tested our method with different kinds of objects. Indeed, the quality of the spherical harmonic description of the object depends both on the number of spheres and on the bandwidth bw. To simplify the calculations, we normalize the models and translate them so that the centers of masses coincide with the origin. The spheres are chosen to be equispaced. We can exploit an interesting property offered by our decomposition. When the radius of the sphere changes and the intersection of the tetrahedron $H$ and the sphere is always as depicted in figure 1(d) then the spherical harmonic coefficients associated to $H$ remain the same. Figure 8 and 9 show a reconstruction of different kind of models using different spheres radii and bandwidths.

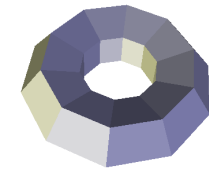

(a) original

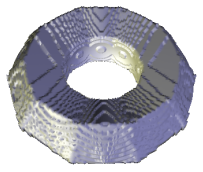

(b) 50 spheres

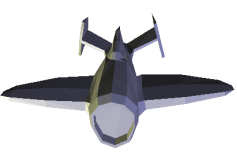

(c) original

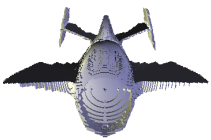

(d) 100 spheres

Figure 8: reconstruction of a polygonized torus and aircraft using $b w=64$

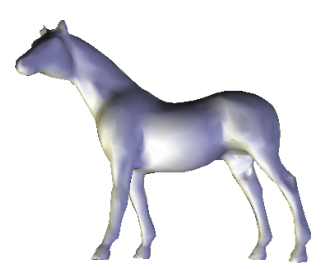

(a) original

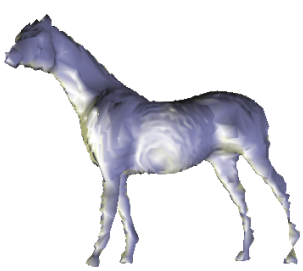

(b) 100 spheres

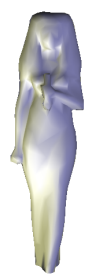

(c) original

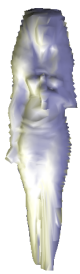

(d) 100 spheres

Figure 9: reconstruction of a horse and Isis statue using $b w=64$

\section{Conclusion}

The proposed method allows us to compute the spherical harmonic coefficients directly from any closed triangulated mesh. The integrations performed over a single triangle are independent of the other triangles. Therefore the calculation can be parallelized without any overlapping problem.

For more information about the code, a web site is now available at http://liris.cnrs.fr/mmousa/. 


\section{Acknowledgement}

Special thanks to the two reviewers of JGT for their help and support to improve the appearence and structure of this paper. In fact, they provide us with valuable comments and suggestions.

\section{References}

[1] W. E. Byerly. Spherical Harmonics, chapter 6, pages 195-218. New York: Dover, 1959. An Elementary Treatise on Fourier's Series, and Spherical, Cylindrical, and Ellipsoidal Harmonics, with Applications to Problems in Mathematical Physics.

[2] M. Galassi et al. GNU Scientific Library Reference Manual (2nd Ed.). http://www.gnu.org/software/gsl/.

[3] T. Funkhouser, P. Min, M. Kazhdan, J. Chen, A. Halderman, D. Dobkin, and D. Jacobs. A search engine for 3d models. ACM Transactions on Graphics, 22(1):83-105, January 2003.

[4] E. W. Hobson. The Theory of Spherical and Ellipsoidal Harmonics. New York: Chelsea, 1955.

[5] A. Kaufman and E. Shimony. 3d scan-conversion algorithms for voxel-based graphics. In SI3D '86: Proceedings of the 1986 workshop on Interactive 3D graphics, pages 45-75, New York, NY, USA, 1987. ACM Press.

[6] M. Kazhdan, T. Funkhouser, and S. Rusinkiewicz. Rotation invariant spherical harmonic representation of 3d shape descriptors. In SGP '03: Proceedings of the Eurographics/ACM SIGGRAPH symposium on Geometry processing, pages 156-164. Eurographics Association, 2003.

[7] D. Saupe and D. V. Vranic. 3d model retrieval with spherical harmonics and moments. In Proceedings of the 23rd DAGM-Symposium on Pattern Recognition, pages 392-397. Springer-Verlag, September 2001.

[8] D. V. Vranic and D. Saupe. Description of 3d-shape using a complex function on the sphere. In ICME '02: Proceedings of the IEEE International Conference on Multimedia and Expo, pages 177$180,2002$.

[9] Eric W. Weisstein. Legendre Polynomial. From MathWorld : A Wolfram Web Resource. http://mathworld.wolfram.com/LegendrePolynomial.html.

[10] C. Zhang and T. Chen. Efficient feature extraction for $2 \mathrm{~d} / 3 \mathrm{~d}$ objects in mesh representation. In ICIP '01: Proceedings of the International Conference on Image Processing, pages 935-938, October 2001. 